\title{
Learning from Pokémon GO and Preparing for Potter: The Impact of Augmented Reality Video Games on Adolescent Physical Activity
}

\author{
Laura McLaughlin BA, Prithwijit Das MSEd, Kate Fruitman BA and Ruth Milanaik DO* \\ Department of Developmental and Behavioral Pediatrics, Steven and Alexandra Cohen Children's Medical \\ Center, USA
}

*Corresponding author: Ruth Milanaik, Department of Developmental and Behavioral Pediatrics, Cohen Children's Medical Center of New York, 1983 Marcus Avenue Suite 130, Lake Success, NY 11042, USA, Tel: 516-802-6100

\begin{abstract}
Background: Only $25 \%$ of US high school students meet CDC physical activity guidelines. Over $40 \%$ report playing video games for $>3$ hours/day. This epidemic of inactivity may lead to health consequences including obesity. Augmented Reality Games (ARGs) may increase activity through video gameplay. Our objective was to quantify the effect of an ARG, Pokémon GO, on adolescent physical activity.

Methods: Parents $\left(n_{e}=546\right)$ of children ages 13-18 completed an anonymous online questionnaire about their child's physical activity and Pokémon GO gameplay. Subjects recorded the number of steps taken by their child for 3 weeks; Week 1 preceded the game's release, Week 2 was immediately after release, and Week 3,1 month after. The dataset consisted of 2 groups: those who started playing around Pokémon GO's release (Pokémon GO Group, $n=418$ ) and those who did not play or started playing after Week 1 (Comparison Group, $n=128$ ). Median daily steps of each week were compared between groups.

Results: There were no significant differences in age, gender, ethnicity or income between groups. The Comparison Group had a $14.8 \%$ increase in steps between Weeks 1 and 2; the Pokémon GO Group had a 29.1\% increase in that same time. Weeks 1 and 3 were not significantly different between the two groups but Week 2 was $(p>0.05)$.

Conclusions: Pokémon GO led to a minimal but significant initial increase in activity. Although future studies are needed to determine the long-term effects of ARGs on physical activity, our study suggests that the purported exercise benefits are minimal.
\end{abstract}

\section{Keywords}

Augmented reality video games, Physical activity, Obesity

\section{Background}

Obesity is one of the most pressing public health concerns for today's adolescents. The Center for Disease Control's (CDC) Youth Risk Behavior Survey conducted in 2015 showed that $29.9 \%$ of adolescents are overweight or obese, meaning they have a body mass index greater than the $85^{\text {th }}$ percentile [1]. The dramatic increase in obesity worldwide can be attributed to several factors, including dietary changes, physical inactivity and the environment [2-5].

Although exercise can reduce the negative outcomes of obesity, only one quarter of high school students meet the CDC's Physical Activity Guidelines, with a concerning $14.3 \%$ of students reporting less than sixty minutes of physical activity per week [1]. Video games are cited as a cause of the rise in obesity prevalence because many children spend large amounts of time stationary instead of playing outdoors and exercising [6]. In fact, the conventional video game format has been independently linked to hypertension and obesity in numerous studies $[7,8]$. Despite efforts to discourage video gaming and encourage physical activity, the number of children playing video or computer games for three or more hours per day has only increased [1,9].

Recently, a new style of video game has become enormously popular: The Augmented Reality Video Game (ARG). ARGs incorporate aspects of the real, tangible world into the world of the video game, where 
users explore the game and the physical environment simultaneously. In July of 2016, Niantic Labs Inc. released Pokémon GO, an ARG based on Pokémon, a popular franchise that produces children's video games, card games, and television shows, initially released by Nintendo in the mid-1990s. Pokémon GO, the ARG, GPS-based version of the game achieved similar success soon after its release, amassing over 21 million users in a week [10]. It continues to have a current active user base of 65 million people each month. Similar to the original video game version, players collect "Pokémon" or animal-like characters and battle the Pokémon against other users. However, in the ARG version, the game map is overlaid onto a real, GPS-based map and the Pokémon appear in real-life locations. Players are physically required to move around in their community to these specific physical landmarks (such as parks, statues, churches, and stores) to catch Pokémon and play against other users. Advancement in the game is contingent on distance travelled.

Due to the enormous popularity of the game, many users reported dramatic increases in physical activity as they walked around their communities searching for Pokémon [11-13]. These anecdotal health claims, proven to be short term in adults, have yet to be substantiated for children [14,15]. As Pokémon was originally designed and intended as a children's game, the impact on childhood activity may be substantially different.

Additionally, Niantic is now preparing to launch, in partnership with Warner Bros. Interactive Entertainment, its next major follow-up ARG to Pokémon GO based on the Harry Potter franchise, tentatively titled Harry Potter: Wizards Unite. Considering the enormous worldwide popularity of the franchise, the new ARG is anticipated to garner a wide fan base of young enthusiastic players [16]. This research study explores the initial and sustained impact of Pokémon GO ARG usage on physical activity levels of children and adolescents.

\section{Methods}

\section{Study design and sample}

An anonymous online questionnaire was developed through Qualtrics, a HIPAA compliant online data collection platform. The questionnaire was made available through Amazon's free Mechanical Turk (MTurk) platform. MTurk allows for the recruitment of a nationally representative population and yields similar reliability to traditional data collection techniques [17]. Potential participants were able to view the questionnaire on MTurk and determine if they were eligible to participate. MTurk subscribers were eligible if they were the parent or guardian of a child between the ages of 13 to 18 years old. The child of the participants must also have had an iPhone $5 \mathrm{~s}$ or newer because these phones have an accelerometer that allows for accurate measurement of steps [18]. This data is stored in the Health data application, which is found on all iPhones and cannot be deleted. Participants must have had access to their child's iPhone in order to report the data. It is impossible to calculate the response rate for the survey as the number of eligible participants who viewed the online posting cannot be determined.

Once participants accessed the survey, they were asked a series of questions about their child's use of Pokémon GO and other video games. Participants indicated if/when their child started playing Pokémon GO and how much they played each day. The questionnaire prompted participants to access the Health data application on their child's iPhone and input the number of steps taken daily for three different weeks during the summer of 2016. The first week (Week 1 ) started on June $30^{\text {th }}$ and ended on July $6^{\text {th }}$, before the release of Pokémon GO. The second week (Week 2) of data collection started a few days after the game's release on July $11^{\text {th }}$ and continued through July $17^{\text {th }}$. Week 3 was the week before the release of the survey, starting August $10^{\text {th }}$ and continuing through August $16^{\text {th }}$. In this study, the number of steps taken each day was used as a proxy for level of physical activity. Participants responded to general demographic questions about themselves and their child, including age, gender, race, and ethnicity.

\section{Statistical analysis}

Incomplete responses were removed from the data set. Additionally, if the indicated number of steps for any days was too low (less than 50) or too high (more than 70,000), then the observation was removed from the data set. These points were eliminated to prevent any outliers from significantly altering the daily averages for each week. The median number of daily steps was calculated for each of the three weeks, and the number of daily steps in each week was compared using a paired, non-parametric Wilcoxon test. Subsequently, the data were split into two different groups. The first group (the Comparison Group) consisted of those who did not play Pokémon GO and those who started playing after Week 2 of data collection. The second group (Pokémon GO Group) contained participants who indicated that their child started playing Pokémon GO within three weeks of its release, when it was most popular. The number of steps taken by children who do not play Pokémon GO or who started playing after Week 2 of data collection were compared to those of the Pokémon GO group using an unpaired, non-parametric Wilcoxon test. If Pokémon GO does increase physical activity, then those who started playing earlier would have an expected increase in daily step count between Weeks 1 and 2. Those who do not play or started playing after Week 2 would not have this projected increase during the same time period. This Comparison Group will help eliminate potential confounding factors that 
Table 1: Median Parent-Reported steps taken daily during the summer of 2016. Week 1 precedes release of Pokemon Go; Weeks 2 and 3 follow the release.

\begin{tabular}{|l|l|l|l|}
\hline Week & Overall Group & Comparison Group & Pokemon Group \\
\hline 1 & 3173.5 & $3,163.5$ & $3,174.5$ \\
\hline 2 & 4,007 & $3,631.0$ & $4,097.5$ \\
\hline 3 & $3,933.5$ & $3,967.0$ & $3,924.5$ \\
\hline
\end{tabular}

Table 2: Change in Median Daily Steps.

\begin{tabular}{|l|l|l|l|}
\hline Week & Overall Group, n (\%) & Comparison Group, n (\%) & Pokemon Group, n (\%) \\
\hline Week 1 to Week 2 & $883.5(26.3 \%)$ & $467.5(14.8 \%)$ & $923.0(29.1 \%)$ \\
\hline Week 2 to Week 3 & $-73(-1.8 \%)$ & $336.0(9.3 \%)$ & $-173.0(-4.2 \%)$ \\
\hline
\end{tabular}

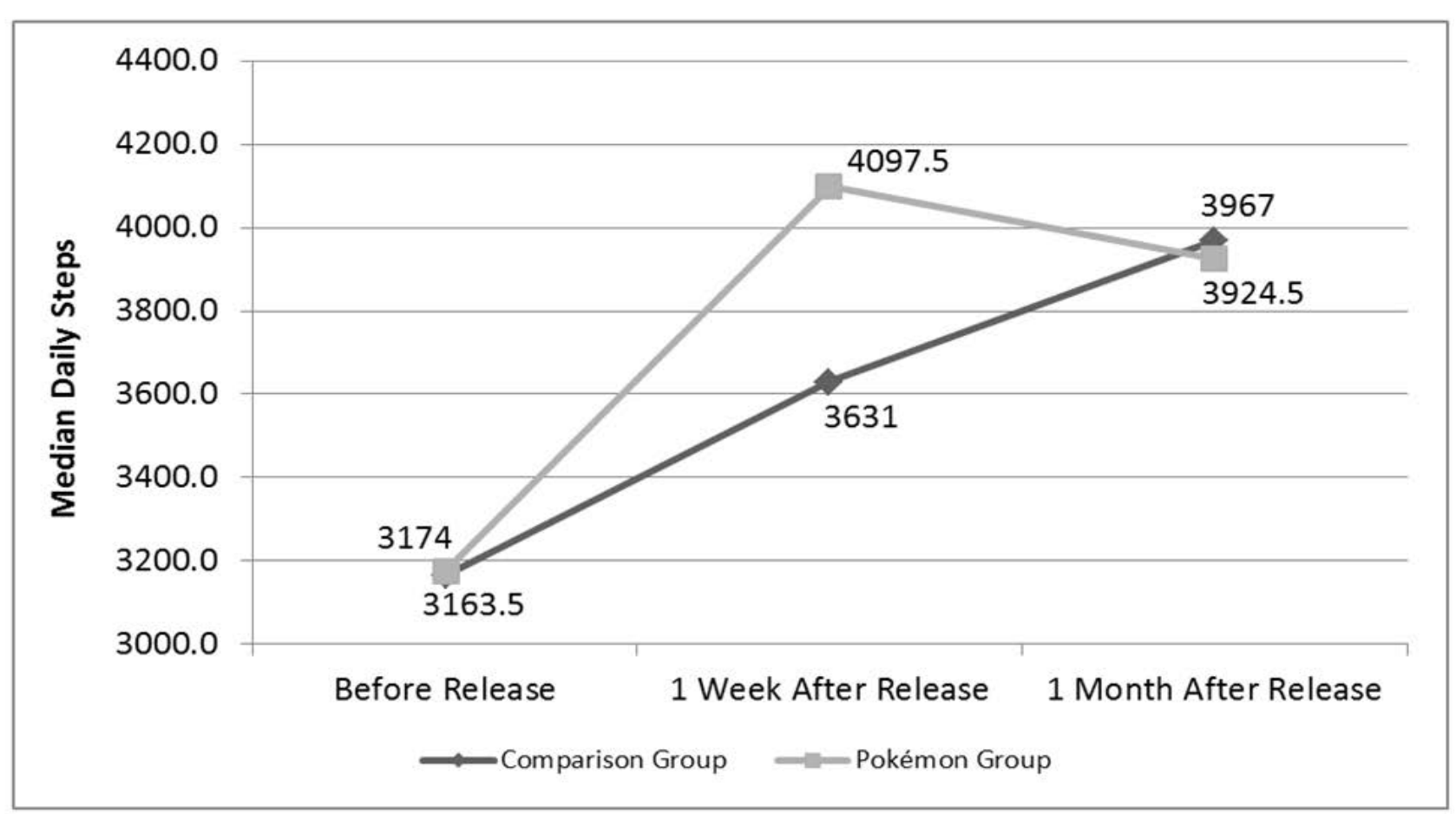

Figure 1: Median parent-reported daily steps taken throughout the summer.

could cause changes in number of steps throughout the summer. Demographic information, including age, race, gender, and income, were given coded numerical values to facilitate statistical analysis.

\section{Results}

Nearly fifteen hundred people completed the study ( $n=1,443$ ), but after eliminating incomplete responses and responses that contained unreasonable step counts, 546 responses were eligible for analysis $\left(n_{e}=546\right)$. Twothirds of the respondents' children were male ( $n=$ 364). Although this gender ratio differs from that of the general population, it is congruent with the Pokémon player user base, which consists primarily of young boys. The average age of the respondents' children was 14.7 years old.

In the overall data set, the median number of daily steps taken was 3,173.5 steps per day during Week 1 (Table 1). During Week 2, after the release of Pokémon GO, participants' children took a median of $4,007.0$ steps per day and during Week 3 , they took a median of $3,933.5$ steps per day. The number of daily steps was not normally distributed (Shapiro test, $\mathrm{p}<0.01$ ). To track the change in number of daily steps for each individual, the weeks were compared using a paired, non-parametric Wilcoxon test. Among the children, there was an 883.5 step (26.3\%) increase in steps between Weeks 1 and 2 (paired Wilcoxon test, $\mathrm{p}<0.01$ ), but there was no significant change in steps between Weeks 2 and 3 (paired Wilcoxon test, $\mathrm{p}=0.75$ ) (Table 2).

The dataset was subsequently reorganized into two different groups: Those who started playing around the game's release (Pokémon GO Group, $n=418$ ) and those who do not play or started playing in the week or two before the survey (Comparison Group, $n=128$ ). There were no significant differences in age, gender, ethnicity or income between the two groups. The weekly values were compared between groups using an unpaired, non-parametric Wilcoxon test. The median number of steps during the first week for the Pokémon GO Group was 3,174.5 steps; median steps during the same time period for the Comparison Group was 3,163.5 steps (unpaired Wilcoxon test: $p=0.20$ ). During Week 2 , the Pokémon GO Group took 4,097.5 steps per day, and the 
Comparison Group took 3,631 steps per day (unpaired Wilcoxon test: $p=0.039$ ). Finally, during Week 3 , the Pokémon GO Group took 3,924.5 steps, while the Comparison Group took 3,967.0 steps per day (unpaired Wilcoxon test: $\mathrm{p}=0.53$ ) (Figure 1 ).

The Comparison Group had a 467.5 step (14.8\%) increase in steps between Weeks 1 and 2 (paired Wilcoxon test, $\mathrm{p}<0.01$ ) and the Pokémon GO Group had a 923.0 step (29.1\%) increase in daily steps over that same time period (paired Wilcoxon test, $p<0.01$ ). Neither group had significant differences in steps between Weeks 2 and 3 (paired Wilcoxon test, $p>0.26$ ).

\section{Discussion}

All groups exhibited significant increases in number of steps taken throughout the summer. The largest increase occurred between Weeks 1 and 2, at the release of Pokémon GO. The $26.3 \%$ increase in steps during this time can be explained by the many factors that influence a child's activity level during summer, including the end of the school year, summer camps, vacation activities, sports practice, etc. However, both the Comparison and Pokémon GO Groups were subject to similar confounding factors, yet the Pokémon GO Group had an increase in average number of daily steps that was twice that of the Comparison Group. Consequently, it can be inferred that playing Pokémon GO led to a minimal, but significant increase in activity. Similar findings were reported for adults playing Pokémon GO [19,20].

The moderate decrease in steps between Weeks 2 and 3 for the Pokémon GO Group was not significant, suggesting that participation in the game continued at least past the initial excitement phase, but playing has a ceiling effect on physical activity. A previous study of the impact on Pokémon GO on physical activity in adults indicates that the increase in steps subsided after a month [19]. The Comparison Group had a larger increase in steps than the Pokémon GO Group between Weeks 2 and 3. A large portion of this group started playing Pokémon GO during this time frame; however, the small sample of participants who started playing Pokémon GO after Week 2 of data collection was too small for significant statistical analysis.

Despite the continued interest in playing the ARG, change in physical activity was minimal and unlikely to lead to long-term changes in adolescent health. Even if the increase in physical activity due to playing ARGs is not dramatic, there could be an additional benefit from decreased time spent on sedentary activities, including watching television or playing stationary video games. Studies have shown that excessive sedentary behavior causes poor metabolic health even when physical activity guidelines are met, and obesity and metabolic disease have been causally linked to both decreased physical activity and increased sedentary behavior [2123]. Further research is required to determine whether
Pokémon GO and other ARGs can replace sedentary activities such as video games or more physically active ones like sports or outdoor play.

Additionally, it should be noted that playing an ARG carries concerning safety risks for children. The game serves as a dangerous distraction while walking, crossing streets, and especially driving [24,25]. Furthermore, due to the interactive nature of Pokémon GO, children might be encouraged to interact with other strangers playing the game. The "Lure" function permits players to attract people to isolated locations, making children more vulnerable to criminal activity.

The popularity of Pokémon GO can also be used to the detriment of children's health. In July of 2016, Niantic Labs Inc. partnered with McDonald's in Japan by creating "Poké stops" at store locations [26]. Players can buy in-game items at these Poké Stops, encouraging them to enter McDonald's stores and potentially purchase fast food items. Although such a partnership has yet to occur in United States, it remains a possibility. Informally, stores can attract players by paying a small fee to have a "Lure module" of the game placed in their store. Pokémon GO can conceivably alter consumer behavior by making certain stores and restaurants desirable game locations. Such a strategy could lead to the increased consumption of fast food by vulnerable adolescents and counteract the minimal health benefits of playing the game.

These safety concerns in conjunction with the minimal increase in physical activity indicate that ARGs should not be encouraged as a replacement for typical outdoor activities or lauded as the solution to the country's epidemic of physical inactivity. However, considering the enormous popularity of this ARG with adolescents, additional research is necessary to see if different ARG formats could indeed produce sustained results. As the 2018 expected launch date for Harry Potter; Wizards Unite approaches, all efforts should be made for organizations to partner with Niantic Labs Inc. and Warner Bros. Interactive to ensure the safety of all players and to initiate research projects that may encourage a consistent and sustained increase in daily steps. Furthermore, although Pokémon GO data does not reflect this sustainable increase in exercise, it does indicate the potential to encourage children to engage in physical activity during video game play.

The method used to measure physical activity in this study has certain limitations. First, unlike wearable pedometers, cell-phones only track steps taken while carried. Children might not be able to carry their phones with them at school, summer camp, or sports practice, so the number of daily steps is most likely higher than the ones reported here. However, this study does not claim to report the number of steps taken each day, but rather seeks to compare steps recorded by the phones before and after playing Pokémon GO. The 
increase in steps observed in this study might be a result of adolescents carrying their phones with them for a higher percentage of the day because they are playing Pokémon GO. The increase in number of steps might not be a result of an increase in physical activity, but rather an increase in the percentage of steps that are recorded by the iPhone. Participants were informed that this study was related to Pokémon GO, so there may have been reporting bias. Finally, our sample was limited to iPhone users on MTurk, therefore the generalizability of these findings may be limited.

\section{Conclusion}

The anecdotal health benefits of the enormously popular ARG Pokémon GO were found to be minimal in an adolescent population. Despite these seemingly unremarkable findings, the movement initiative inspired by Pokémon GO has potential to be more effective if developed further in collaboration with children's health organizations. In advance of the launch of a possibly more influential and child-based planned game, all efforts should be made to encourage families to play safely and not replace typical childhood exercise with gameplay.

\section{Funding Source}

No funding was secured for this study.

\section{Acknowledgements}

N/A.

\section{Author Disclosure Statement}

No competing financial interests exist.

\section{Conflict of Interest}

The authors have no conflicts of interest to disclose.

\section{References}

1. Centers for Disease Control and Prevention (2017) 2015 Youth Risk Behavior Survey Data.

2. Chung WK, Leibel RL (2008) Considerations regarding the genetics of obesity. Obesity (Silver Spring) 16: S33-S39.

3. Aaron DJ, Storti KL, Robertson RJ, Kriska AM, LaPorte RE (2002) Longitudinal study of the number and choice of leisure time physical activities from mid to late adolescence: implications for school curricula and community recreation programs. Arch Pediatr Adolesc Med 156: 1075-1080.

4. Jahns L, Siega-Riz AM, Popkin BM (2001) The increasing prevalence of snacking among US children from 1977 to 1996. J Pediatr 138: 493-498.

5. McConahy KL, Smiciklas-Wright $\mathrm{H}$, Mitchell DC, Picciano MF (2004) Portion size of common foods predicts energy intake among preschool-aged children. J Am Diet Assoc 104: $975-979$.

6. Reilly JJ, Methven E, McDowell ZC, Hacking B, Alexander D, et al. (2003) Health consequences of obesity. Arch Dis Child 88: 748-752.

7. Centers for Disease Control and Prevention (CDC) (2017)
Childhood Obesity Causes and Consequences.

8. Lakshman R, Elks CE, Ong KK (2012) Childhood obesity. Circulation 126: 1770-1779.

9. Dietz W (1998) Health Consequences of Obesity in Youth: Childhood Predictors of Adult Disease. Pediatrics 101: 518525.

10. Biro F, Wien M (2010) Childhood obesity and adult morbidities. Am J Clin Nutr 91: 1499-1505.

11. Sisson SB, Broyles ST, Baker BL, Katzmarzyk PT (2010) Screen Time, Physical Activity, and Overweight in U.S. Youth: National Survey of Children's Health 2003. J Adolesc Health 47: 309-311.

12. Goldfield GS, Kenny GP, Hadjiyannakis S, Phillips P, Alberga AS, et al. (2011) Video game playing is independently associated with blood pressure and lipids in overweight and obese adolescents. PLoS One 6: e26643.

13. Foley L, Maddison R (2010) Use of Active Video Games to Increase Physical Activity in Children: A (Virtual) Reality? Pediatr Exerc Sci 22: 7-20.

14. Centers for Disease Control and Prevention (2013) Youth Risk Behavior Survey Data.

15. Metcalf B, Henley W, Wilkin T (2012) Effectiveness of intervention on physical activity of children: systematic review and meta-analysis of controlled trials with objectively measured outcomes. BMJ 345: 5888.

16. The Magic of Harry Potter is Coming to a Neighborhood Near You (2017) Niantic.

17. Buhrmester M, Kwang T, Gosling SD (2011) Amazon's Mechanical Turk: A New Source of Inexpensive, Yet HighQuality, Data? Perspect Psychol Sci 6: 3-5.

18. Case MA, Burwick HA, Volpp KG, Patel MS (2015) Accuracy of smartphone applications and wearable devices for tracking physical activity data. JAMA 313: 625-626.

19. Howe KB, Suharlim C, Ueda P, Howe D, Kawachi I, et al. (2016) Gotta catch'em all! Pokémon GO and physical activity among young adults: difference in differences study. BMJ 355: 6270.

20. Althoff T, White RW, Horvitz E (2016) Influence of Pokémon Go on Physical Activity: Study and Implications. J Med Internet Res 18: 315

21. Owen N, Healy G, Matthews C, Dunstan D (2010) Too Much Sitting: The Population-health science of sedentary behavior. Exerc Sport Sci Rev 38: 105-113.

22. Epstein LH, Paluch RA, Gordy CC, Dorn J (2000) Decreasing sedentary behaviors in treating pediatric obesity. Arch Pediatr Adolesc Med 154: 220-226.

23. Hamilton MT, Hamilton DG, Zderic TW (2007) Role of low energy expenditure and sitting in obesity, metabolic syndrome, type 2 diabetes, and cardiovascular disease. Diabetes 56: 2655-2667.

24. Serino M, Cordrey K, McLaughlin L, Milanaik RL (2016) Pokémon GO and augmented virtual reality games: a cautionary commentary for parents and pediatricians. Curr Opin Pediatr 28: 673-677.

25. Ayers JW, Leas EC, Dredze M, Allem JP, Grabowski JG, et al. (2016) Pokémon GO-A New Distraction for Drivers and Pedestrians. JAMA Intern Med 176: 1865-1866.

26. Olson P (2016) Pokémon GO's McDonald's Partnership Points to a Promising Business Model. Forbes. 